\title{
A Study on the Clinical Profile of Patients with Ulcerative Colitis Attending a Tertiary Care Hospital in Central Kerala
}

\author{
Dr. Renny Issac ${ }^{1}$, Dr. Vinu Thomas ${ }^{2}$, Dr. A. Amar Jayanthi ${ }^{3}$ \\ ${ }^{1}$ Assistant professor, \\ ${ }^{2}$ Professor, General Medicine Department, \\ ${ }^{3}$ Associate professor, Anatomy Department \\ Government Medical College, Thrissur, Kerala, India
}

\begin{abstract}
Ulcerative colitis is a chronic disease of the bowel that results in inflammation and ulceration of the colon and rectum. It presents with loose stools mixed with mucous and blood. The exact etiology is unknown. The factors considered frequently are life style changes, smoking, food habits, sanitation, exposure to infections, occupation, disordered immune response and genetic factors. The incidence in Indians is rising. Considering the varied clinical profile in different geographical distribution, it is worth investigating the clinical profile. Indian studies are few in number.Thisresearch is aimed to study the clinical profile of patients with ulcerative colitis attending a tertiary care hospital in central Kerala. A descriptive study conducted in all consecutive patients having a diagnosis of ulcerative colitis attending the medicine, surgery and gastroenterology department was done. The most common clinical feature was loose stools with blood and mucus, with moderate severity and the duration of ranged from 1 month to 7 years. The mean age of onset of symptoms were 40 yrs. Life style modifications including exercise and stress relieving therapies may help reducing the incidence.

Keywords: clinical profile, Diarrhea, Inflammatory Bowel disease, Rectalbleeding, Ulcerative colitis
\end{abstract}

\section{Introduction}

Ulcerative colitis(UC) is a chronic disease of the bowel that results in inflammation and ulceration of the colon and rectum.Ulcerative colitis and Crohns disease, the two components of Irritable bowel syndrome(IBD), has been a problem in industrial-urbanized societies. The incidence and prevalence in developing countries is steadily rising and has been attributed to the Westernized lifestyle of the population[1].A population based study from Punjab found a crude incidence rate of $6.02 / 10^{5}$ per year and prevalence rate of $44.3 / 10^{5}$ inhabitants[2]. The incidence in Indians is also rising. UC presents with diarrhea mixed with blood and mucous. The exact etiology is unknown. The factors considered frequently are life style changes, smoking, food habits, sanitation, exposure to infections, occupation, disordered immune response and genetic factors. The disease occurs in family members,siblings and twins. Molecular genetic studies in IBD, resulted in the identification of nucleotide-binding oligomerization domain containing 2 (NOD2) gene as a risk factor[3]. The incidence varies within different geographical areas. Considering the varied clinical profile in different geographical distribution, it is worth investigating the clinical profile. This sort of investigation has not been undertaken in this part of the world previously. The aim of the present study is to observe the clinical profile of patients with ulcerative colitis attending a tertiary care hospital in central Kerala.

\section{Materials And Methods}

A descriptive study was conducted in a tertiary care hospital in central Kerala from September 2010 to January 2012. All consecutive patients having a diagnosis of ulcerative colitis attending the medicine and gastroenterology departments of Government Medical college Thrissur, were included in this study. Those who declined the consent and those who cannot be followed up were excluded. 35 Patients with a diagnosis of UC from the department of Medicine and Gastroenterology were enrolled to the study after written informed consent. Patients demographic data, physical examination findings, laboratory findings and endoscopic findings are noted in the specified proforma. Statistical analysis were performed by using appropriate tests in the software SPSS version 18. All variables including the qualitative and quantitative were documented and assigned to specific numerical code and entered in the excel sheet and imported to the software and some variables are defined and analyzed. The means and proportions were computed for background variables.

\section{Results And Discussion}

Ulcerative colitis is a multifactorial genetic disorder that produce changes in the mucosal lining or an abnormal immune response to normal bacteria in the gastro intestinal tract. During this study period a total of 35 patients(male 21 and female 14) were registered with ulcerative colitis, the age ranging from 19yrsto 79yrs. The mean age of onset was 40years. Similar finding was reported by Loftus EVetal [4]where the patients had the 
disease between 15 and 30 years, with the mean age being 40 years.(Table 1) In this study the male female ratio was1.5:1.

\subsection{Clinical presentation}

Present study observes all the patients had loose stools and 33 out of 35 had a frequency of 4-6 per day.(Table 2) The onset of UC typically is slow and insidious. Ulcerative Colitis presented with loose stools, blood and mucus, the duration of which ranged from 1 month to 7 years. The present study observes a mean delay of diagnosis as 1 year from the onset of symptoms, especially loose stools. Pimentel M et al[5] observed the prodromal period as $1.2+/-1.8$ year for ulcerative colitis. Majority had obvious blood in stool (77\%) and only $22 \%$ had streaky blood mixed with mucus as the initial presentation. Rao SS et al[6] has observed that rectal bleeding is common in UC. The characteristic of the bleeding is determined by the location of the disease. Patients with proctitis usually complain of passing fresh blood, either separately from the stool or streaked on the surface of a normal or hard stool.

\subsection{Associated symptoms}

The present study reports that $28 \%$ of patients had abdominal pain as a prominent symptom. Many patients with UC complain of abdominal pain with active disease, but pain generally is not a prominent symptom. Patients may experience vague lower abdominal discomfort, an ache in the left iliac fossa, or intermittent abdominal cramping preceding bowel movements and often persisting transiently after defecation. Fever was present in 4 patients $(11.4 \%)$.

\subsection{Severity of the disease}

According to Truelove Witts classification[7], Severity of disease was categorized as moderate in $22(62.9 \%)$, severe in $9(25.7 \%)$ and as mild in $4(11.4 \%)$ patients.

\subsection{Socioeconomic profile}

Out of the 35 patients, $25 \%$ of patients were from the low socio economic status and others belong to middle class. Majority of the female patients were home makers(98\%) and the rest were workers. Most of the male patients were doing business.

\subsection{Environmental risk factors}

In our study only 2 out of 35 patients were smokers, all others were non smokers. Multiple studies consistently have shown that UC is more common among nonsmokers than among current smokers, with the relative risk of UC in nonsmokers ranging from 2 to 6[8]. In Caucasian populations smoking has an opposite effect on UC with odds ratio 0.58 [9]. Case control studies confirm that the relative risk of colitis in ex-smokers is greater than life-long non-smokers, with reduced risk in current smokers[10]. History of occasional consumption of alcohol was given by $4(11.4 \%)$ patients.

\subsection{Co morbidity}

Among the 35 patients one each had primary sclerosing cholangitis, ankylosing spondylitis, and adenocarcinoma of colon. All of these entities are well known associations of ulcerative colitis[11,12]. Habior and co-workers described the association of primary sclerosing cholangitis and ulcerative colitis in two sisters,indicating a genetic predisposition also for this disease[13]. One patient each had cutaneous manifestations like pyoderma gangrenosum and Icthyosis.[14,15]. This study revealed that two patients had undergone hemi colectomy for adenocarcinoma of colon and ilio-cecal tuberculosis. Another patient had hemorrhoids for which surgery was done. As for smoking studies have demonstrated a negative association between appendicectomy and subsequent development of colitis. There were 2 patients who had undergone appendicectomy, in the past for acute appendicitis. Three each of the UC patients presented with other systemic diseases like hypertension, ischemic heart disease, joint pain and one asthma. 40\% had weight loss at presentation. There were 2 patients who had undergone anti tuberculous treatment in the past.

\subsection{General examination findings}

Mean BMI was 21.99 ranging from 17.3 to 27.8, Clubbing was present in 2 patients. 13 out of 35 patients (37.1\%) had pallor.

\subsection{Laboratory findings}

Stool examination revealed blood in all 35 patients. Blood in stool in UC are nonspecific and reflect the severity of the underlying disease. Patients with active proctitis and proctosigmoiditis often have normal laboratory test results. Patients with limited distal disease often pass visible blood in the stool, but the amount of 
blood loss typically is small and anemia, if present, is mild. Patients with active extensive disease or severe distal disease may demonstrate laboratory abnormalities. Hematologic changes, including anemia, leukocytosis and thrombocytosis, reflect active disease. In contrast, patients with quiescent UC typically manifest no laboratory abnormalities.

\section{Conclusion}

This study was able to bring data to the existing profile data of UC patients. Data on the relationship between alcoholism, physical activity, stress, diabetis mellitus, hypertension, asthma, tuberculosis and its treatment drugs are scanty and more studies are needed. There are also some limitations to this study. There is increased risk of colorectal cancer for patients with ulcerative colitis. In conclusion, this study validates the profile of UC patients attending a tertiary care hospital, which is a referral hospital. Therefore the number of cases attending this center does not represent the actual figure in the population. More studies including genomic as well as immunological studies should come out from the primary health care center level.

Table 1. Showing Age Distribution In Ulcerative Colitis Patients

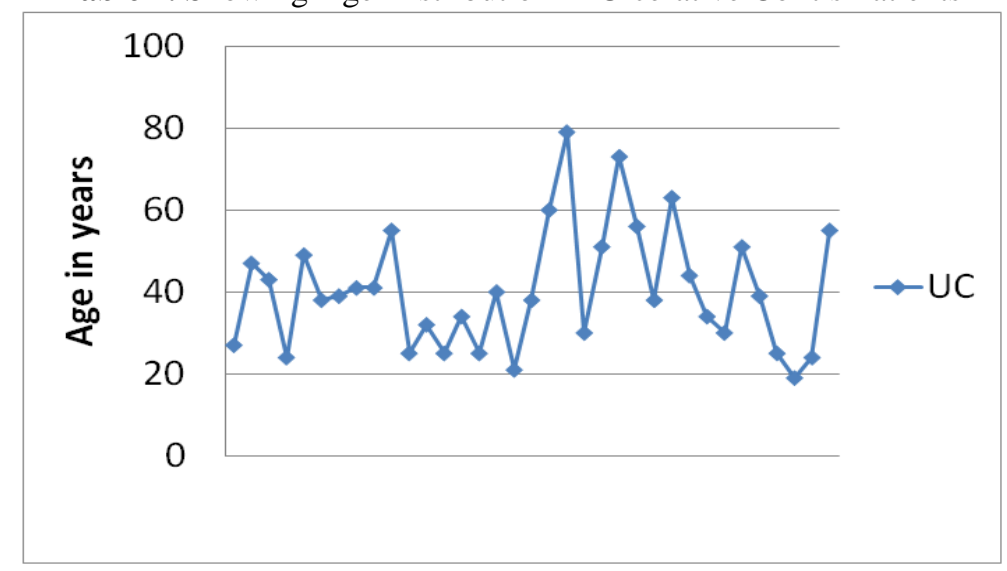

Table 2. Showing Frequency Of Loose Stools In Ulcerative Colitis

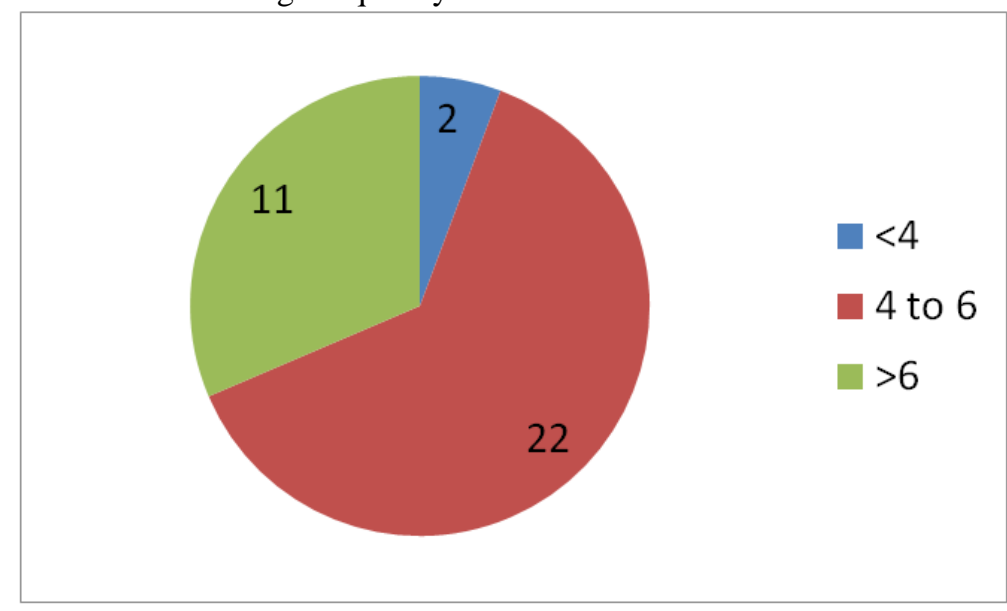

\section{References}

[1]. Amosy E. M'Koma. Inflammatory Bowel Disease: An Expanding Global Health Problem. Clinical Medicine Insights: Gastroenterology. 2013; 6:33-47

[2]. A Sood, V Midha, N Sood, AS Bhatia, G Avasthi. Incidence and prevalence of ulcerative colitis in Punjab, North India.Gut.2003; 52:1587- 1590

[3]. Weronica E. Eka, Mauro Amatoa, Jonas Halfvarsonb. The history of genetics in inflammatory bowel disease. Annals of Gastroenterology.2014;27:294-303.

[4]. Loftus EV: clinical epidemiology of inflammatory bowel disease: incidence, prevalence,and environmental influences. Gastroenterology 126: 1504,2004 ( PMID: 15168363)

[5]. Pimental M, chang M, Chow EJ, Tabibzadeh S, Kirit-Kiriak V, Targan SR, Lin HC.Identification of a prodromal period in Crohn's disease but not ulcerative colitis. Am J Gastroenterol.2000 Dec; 95(12): 3458 - 62.

[6]. Rao SS, Holdsworth CD, Read NW: Symptoms and stool patterns in patients with ulcerative colitis. Gut 1988; $29: 342$.

[7]. Truelove SC, Richards WC: Biopsy studies in ulcerative coitis. BMJ. 1956; 4979: 1315.

[8]. Boyko EJ, Perera DR, KoepsellTD,.Effects of cigarette smoking on the clinical course of ulcerative colitis.Scand J Gastroenterol. 1988; 23: 1147. 
[9]. Sonia Friedman, Richard S, Blumberg.Inflammatory bowel disease.Harrison's Principles of internal medicine. 19 ${ }^{\text {th }}$ edition. 1947.available at www.accessmedicine.com.

[10]. Gareth A O Thomas, John Rhodes, John T Green, Charles Richardson. Role of smoking in irritable bowel syndrome: implications for therapy. Postgrad Med J. 2000;76:273-279.

[11]. Orchard T R, Wordsworth BP, Jewell DP. Peripheral arthropathies in inflammatory bowel disease: their articular distribution and natural history. Gut.1998; 42:387.

[12]. Blackstone MO, Riddell RH, Rogers BH et al. Dysplasia associated lesion or mass(DALM) detected by colonoscopy in longstanding ulcerative colitis: An indication for colectomy. Gastrenterology 1981; 80:366.

[13]. Habior A, Rawa T, Orlowska J, Sankowska M, Lewartowska A, Tilszer A, Pawlak J, Krawczyk m, Buruk E. Association of primary sclerosing cholangitis, ulcerative colitis and coeliac disease in female siblings.Eur J GastroenterolHepatol. 2002 jul; 14(7): 787-91. PMID. 12169991

[14]. Menaachem Y, GotsmanI.clinical manifestations of pyodermagangrenosum associated with inflammatory bowel disease. Isr. Med. Assoc. J. 2004; 6: 88-90.Pubmed

[15]. Thorrnton J R, Teague R H, Low-Beer T S, Read A E. Pyoderma gangrenosum and ulcerative colitis. Gut.1980; 21: 247-248. 